\title{
Traceability and method validation in a photometry laboratory
}

\author{
O. Pellegrino ${ }^{1, \star}$, C. Pires ${ }^{1}$, and E. Filipe ${ }^{2}$ \\ 1 Laboratório de Fotometria, Radiometria e Radiofrequências, IPQ, Portugal \\ 2 Directora do Departamento de Metrologia, IPQ, Portugal
}

Received: 7 July 2010 / Accepted: 22 September 2010

\begin{abstract}
This article displays how the photometry laboratory of the Portuguese Institute for Quality complies with the measurement traceability and method validation, the technical requirements 5.4 and 5.6 of the ISO/IEC 17025. After the presentation of the existing means and the way the metrological traceability is obtained, a short introduction to the internal validation of the measurement method is displayed for the luminous intensity, the illuminance and the illuminance responsivity.
\end{abstract}

Keywords: Luminous intensity; illuminance; illuminance responsivity; metrological traceability; internal verification of measurement method

\section{Introduction}

The Photometry Laboratory of the Portuguese Institute for Quality (IPQ), the National Metrology Institute (NMI) of Portugal, performs calibrations of secondary, reference or working measurement standards for luminous intensity, and performs calibrations of measuring instruments of illuminance or illuminance responsivity. All these are photometric quantities. The candela (symbol cd), one of the seven base units of the International System of Units (SI), has been the unit of the luminous intensity, $I$, since SI was established in 1960. According to the Bouguer-Lambert's law, the quotient between the luminous intensity of a radiative source and the squared constant distance to the source, $D^{2}$, is an invariant, which is called the illuminance, $E=\frac{I}{D^{2}}$ [1]. The unit of illuminance is the lux (symbol lx) and the latter quantity is measured with illuminance meters. It can also be deduced from photocurrents, $y$, measured by photometers through the illuminance responsivity, $s$, value: $E=\frac{y}{s}$.

In the ISO/IEC 17025 assessments [2], which is regularly carried out, the IPQ photometry laboratory (IPL) has to demonstrate the metrological traceability of the measurement results and the validation of its measurement method, i.e. the requirements 5.6 and 5.4 of the standard, respectively. The calibration certificate of a standard lamp for its luminous intensity displays the measurement result of the luminous intensity when the emitted spectral distribution is equal to the one of the blackbody at the $2856 \mathrm{~K}$ colour temperature, i.e. a source known as the illuminant A [3]. The calibration certificate also indicates the

^ Correspondence: opellegrino@mail.ipq.pt functioning conditions of the lamp: the operating power intensity and, as an indication for its stable functioning, the value of the difference in electrical potential between its terminals. Therefore, values of electrical power are included in the assessment of the measurement results of the photometric measurands. Electrical resistances and multimeters are used for the electric measurements in the IPL. The Bouguer-Lambert's law establishes that the measurements of distances are essential to determine the value of the illuminance. In the IPL, they are performed through a measuring system associated to the photometric bench. Finally, to meet the reference environment conditions in the IPL, which are obtained by a heating, ventilation and air conditioning (HVAC) system, the corresponding measurands are also measured with calibrated thermometer and hygrometer.

In order to assess the performance of the measuring method, in a first approach, internal verifications of the average values and the standard deviations of the photometric measurands are performed. Then variances of reproducibility from average values and repeatability variances can be estimated following the ISO EN 5725-2 standard [4]. In this approach, the so-estimated reproducibility standard deviation is considered as the measurement uncertainty component evaluated by type A evaluation of the measurement uncertainty. On the other hand, the magnitudes of measurement results can be estimated by the observed relative standard uncertainties and the relative variations of the corresponding quantity values. Numerically, they can be compared to the $[0.5 ; 1.0] \%$ relative expanded uncertainty of luminous intensity, at the $k=2$ level, for the calibration of primary standard lamps by other NMI Primary Photometry 


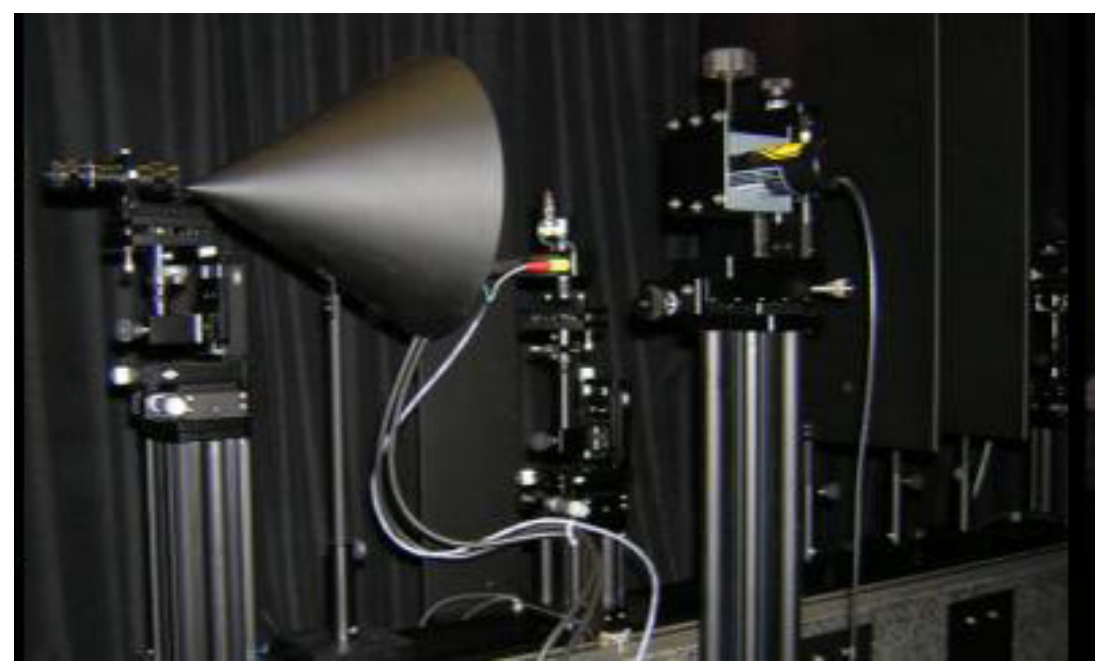

Fig. 1. Photometric bench of the IPQ Photometry Laboratory.

Laboratories [3]. Then, some conclusions about the internal validation of the measurement results of the IPL can be drawn.

\section{Measurement equipment}

The IPL measurement equipment for photometry consists of an almost 4-metre long photometric bench, located in a room with black walls, black curtains, with a temperature and humidity environment controlled by an HVAC system. Its temperature is equal to $(23 \pm 3){ }^{\circ} \mathrm{C}$ and its relative humidity to $(50 \pm 15) \%$. In addition, the photometric bench consists of a rail which position is measured with a digital reading system, with a $50 \mu \mathrm{m}$ standard deviation in reproductibility conditions of measurement. Lamp or detector holders enable to set the position along three perpendicular axis and three angular rotations; they are located at the top of cars moving on the rails of the photometric bench. The electric components of the laboratory are a Bouhnik class $10^{-5}$ stabilised power supply with 10 ppm stability, a 10 ppm stabilized high accuracy electrical resistance with stabilization in temperature and two Agilent 34401A digital multimeters. 3 Osram Wi 41/G tungsten filament lamps, acquired late 2005, is the main primary standard lamp set, 9 Oriel working standard lamps of $50 \mathrm{~W}, 250 \mathrm{~W}$ and $1000 \mathrm{~W}$ power and a LMT P15 F0T photometer is the primary standard photometer of IPL.

In Figure 1, the photometric bench of the IPL experimental setup is displayed. From left to right, one can see the optical alignment system, the conical light trap, the lamp holder, a laser of which the beam materializes the plane of the lamp filament, straight light baffles and the photometer upon its holder.

\section{Measurement procedure}

The filament planes of the reference lamp, $P_{j}$, and of the lamp under calibration, $S_{i, j}$, are located at the same distance from the photometer receiving plane. Therefore, there is proportionality between the respective lamps luminous intensities, $I_{v}$, and the photocurrents, $y$, leading to $[5]$ :

$$
I_{v, S i, j}=\frac{I_{v, P j} y_{S i, j}}{y_{P j}} .
$$

After measuring $n$ replica of the quantity $x$, the average values and standard deviations of repeatability and reproducibility, $m_{x, L, j}, s_{r x, L, j}$ and $s_{R x, L, j}$, (where " $L j$ " stands for the $j$ th replica with lamp $L$ ) respectively, can be deduced. Indeed, the ISO EN 5725-2 standard gives a relationship between these quantities and the standard deviation of the average values, $s_{m x, L, j}$ :

$$
s_{R x, L, j}^{2}=s_{m x, L, j}^{2}+\frac{n-1}{n} s_{r x, L, j}^{2} .
$$

Previously given by the ASTM E691 standard [6], the above relationship enables to estimate the reproducibility standard deviation, which leads to the uncertainty evaluated by the type A evaluation.

Measurement models [5] of the luminous intensity, taking into account the lamp ageing time, $t_{a}$, the calibrated electrical resistance values, $R$, the multimeter calibration coefficients, $\alpha_{m}$, geometrical factors for the orientation of the lamps, $g_{j}$, the reference lamp luminous intensity values, $I_{v, R}$, and the photocurrent measurement values, $y$, were gathered by G. Sauter of PTB, for the "2nd Expert Symposium on Measurement Uncertainty" of the International Commission on Illumination (CIE). These models are used in this work and allow to follow the approach of the Guide to the expression of the Uncertainties Measurements (GUM) [7] to estimate the consequent standard uncertainty associated to the luminous intensities of the lamps under calibration, $I_{v}\left(t_{a}, R, a_{m}, g_{j}, I_{v, R}, y\right)$. In this approach, the respective sensitivity coefficients, $c_{i}=\frac{\partial I_{v}}{\partial x_{i}}$, of the input quantities, $x_{i}$, that are present in the measurement model are calculated. The law of propagation of uncertainty is then applied, considering no correlation between the input quantities [8], leading to the expression 
Table 1. Photocurrent value: $y_{m}$; photocurrent relative standard uncertainty $u_{r}\left(y_{m}\right)$ and relative variation of photocurrent value, for the primary standard lamps serial numbers $a, b$ and $c$, and for the instants $T$ and $T+10 \mathrm{~h}$.

\begin{tabular}{lccc|cccc}
\hline & \multicolumn{3}{c}{$T$} & \multicolumn{4}{c}{$T+10 \mathrm{~h}$} \\
Lamp & & $u\left(y_{m}\right) /$ & $u_{r}\left(y_{m}\right)$ & & $u\left(y_{m}\right) /$ & $u_{r}\left(y_{m}\right) /$ & $\left(\Delta y_{m} / y_{m}\right) /$ \\
& & & & & & & \\
& & & & & \\
$\mathrm{SN}$ & $\left.y_{m} / \mathrm{nA}\right)$ & $y_{m} / \mathrm{nA}$ & $\mathrm{pA}$ & $(\mathrm{fA} / \mathrm{nA})$ & $(\mathrm{pA} / \mathrm{nA})$ \\
\hline$a$ & 1.4431 & 1.2 & 0.83 & 1.4373 & 1.8 & 1.3 & -4.0 \\
$b$ & 1.3915 & 2.1 & 1.5 & 1.3889 & 4.6 & 3.3 & -1.9 \\
$c$ & 1.4695 & 0.1 & 0.07 & 1.4666 & 0.4 & 0.27 & -2.0 \\
\hline
\end{tabular}

of the standard uncertainty of the luminous intensity:

$$
u\left(I_{v, j}\right)=\sqrt{\sum_{i}\left[c_{i} u\left(x_{i}\right)\right]^{2}}
$$

from the standard uncertainties of the input quantities, $u\left(x_{i}\right)$. Whatever the input quantity $x_{i}$, the associated standard uncertainty is the quadratic sum of its type A evaluation and of its type B evaluation, when possible.

Then, for each lamp under calibration, the luminous intensity is determined by applying the same procedure with, at least, one other reference lamp. Finally, the resulting luminous intensity value of the lamp under calibration is equal to the average of the values obtained for all the used reference lamps. The resulting luminous intensity standard uncertainty is the one of average of the luminous intensities obtained from the reference lamps.

Conversely, from the measured photocurrents of the reference lamps used to determine the luminous intensity of lamps, it is possible to estimate the values of the parameters for the internal validation of the measurements results, according to equation (2). This overall procedure was adopted in this work.

\section{Results}

The 3 primary standard lamps and the primary standard photometer of IPL are calibrated at the Primary Photometry Laboratory of the CNAM/LNE, the NMI of France, with a periodicity for the calibration of $25 \mathrm{~h}$. Then, the three sets of secondary standard lamps are calibrated annually with the IPL primary standard lamps and the IPL secondary standard lamps to enable the calibration of other incandescent lamps for luminous intensity. Also the secondary standard lamps enable to calibrate illuminance meters and photometers for illuminance and illuminance responsivity, respectively [9]. As a matter of fact, photometers are calibrated through the reference photometer that is annually calibrated and traceable to SI through the French NMI.

Due to the existence of Primary Metrology Laboratories at the IPQ, the Thermometry and Hygrometry Laboratory, the Laboratory of the Electrical Quantities and the Laboratory of Length, the calibrations of the corresponding measuring instruments used in IPL are regularly performed.
The statements of the previous paragraphs evidence that the measurement results "....can be related to a reference through a documented unbroken chain of calibrations...", which is the definition of the metrological traceability, the point 2.41 of the "International vocabulary of metrology - basic and general concepts and associated terms (VIM)" [10]. In this definition, the term "reference" is a measurement unit of the SI, i.e. the traceability to the SI [11].

Since their acquisition, the primary standard lamps of IPL have been used for about $10 \mathrm{~h}$, from the regular calibration of secondary standard lamps to the participation to an interlaboratory comparison of luminous intensities [12]. The experimental data for having used the three primary standard lamps let us estimate some parameter values for the internal validation of the measurement method. In Photometry Laboratory, the main measurand is the luminous intensity. So the obtained experimental data and the parameter values of interest deduced from the measurement method have to be compared with the luminous intensity relative expanded uncertainties, which are published data [3]. In this work, we consider the values for relative variation of the average of a characteristic quantity, $z_{m}$, and its relative standard uncertainty, $u_{r}\left(z_{m}\right)$. As it enables to determine other photometric quantities, the photocurrent that outputs from the multimeter associated to the illuminated photometer is what we choose as a characteristic quantity. Indeed, from equation (1), the knowledge of $u\left(y_{P j, m}\right)$ and $u_{r}\left(y_{P j, m}\right)$ enables to deduce $u_{r}\left(y_{S i, j, m}\right)$ or $u_{r}\left(I_{v, S j, m}\right)$, according to the measurand. Furthermore, the good stability of the primary standard lamps and the $u_{r}\left(y_{P j, m}\right)$ values being smaller than the other $u_{r}\left(z_{m}\right)$ confirm the choice made for the characteristic photometric quantity leading to the parameter values of the internal validation of the measurement method. In Table 1, the absolute and relative values of the measurands are displayed, where the primary standard lamps are identified by the serial number " $a$ ", " $b$ " and " $c$ ".

From Table 1, it appears that the magnitudes of the relative variation for the photocurrent measurement values, $\left|\frac{\Delta y_{m}}{y_{m}}\right|$, are smaller than the ones of the $[0.25 ; 0.50] \%$ interval. The latter corresponds to the relative standard uncertainty values of some NMIs primary photometry calibration certificates for the luminous intensity [3]. Table 1 also shows the same order of magnitude of the relative standard uncertainty for the photocurrent 
measurement value, $u_{r}\left(y_{P j, m}\right)$, as the one published in reference work [5].

The two previous observations about our results let us conclude that the requirements for internal validation of a measurement method obtained at IPL for photocurrent in photometric quantities measuring system are met. Furthermore, the photometric quantities, due to their respective definitions, illuminance and illuminance responsivity, directly depend on the luminous intensity and their relative uncertainty which is of the same magnitude range as the one of the luminous intensity. Therefore, the internal validation of the measurement results for the measurands can also be asserted from the observations derived from the results of Table 1. Finally, the external validation of the measurement method may be concluded from the final report of the Key Comparison EURAMET.PR-K3.a of luminous intensity, conducted by the PTB Primary Photometry Laboratory, to which IPL participated.

\section{Conclusion}

The metrological traceability of the results is an essential requirement for the quality assurance of a laboratory in metrology. This is performed by the calibration of the measuring instruments used in the IPQ Photometry laboratory. Mainly settled on the repeatability and the reproducibility standard uncertainties, the stable results displayed in this work enable a step forward for the quality of the results obtained by the laboratory, i.e. the internal validation of the measurement method. These results were obtained with photocurrent as photometric quantity from which relative variation and relative standard uncertainty were considered. Due to their close measurement models, the results were extended to other main photometric quantities like luminous intensity, illuminance and illuminance responsivity. According to the result of interlaboratory comparison of luminous intensities, the laboratory may evidence external validation of its measurement method.

\section{References}

1. J.W.T. Walsh, Photometry, 3rd edn. (Constable \& Co. Ltd., 1958)

2. ISO/IEC 17025:2005, General requirements for the competence of testing and calibration laboratories

3. CNAM-INM, Calibration Certificate $\mathrm{n}^{\circ}$ 1139-ra-05, Standard lamp of luminous intensity (2005); Y. Ohno, NIST Special Publication 250-37, NIST measurement Services: Photometric Calibrations (1997)

4. ISO 5725-2:1994, Accuracy (trueness and precision) of measurement methods and results, Part 2: basic method for the determination of repeatability and reproducibility of a standard measurement method

5. G. Sauter, CIE Uncertainty Symposium - Tutorial (2006)

6. ASTM E691-92, Standard Practice for Conducting an Interlaboratory Study to Determine the Precision of a Test Method

7. ISO/IEC Guide 98-3:2008, Guide to the expression of Uncertainty in Measurement (GUM:1995) (published by ISO in the name of BIPM, IEC, IFCC, IUPAC, IUPAP and OIML)

8. ISO/IEC Guide 98-3:2008, Guide to the expression of Uncertainty in Measurement (GUM:1995) (published by ISO in the name of BIPM, IEC, IFCC, IUPAC, IUPAP and OIML), Part 5.1

9. J. Bastié, La Photométrie photoélectrique à l'Institut National de Métrologie du Conservatoire National des Arts et Métiers, Bulletin BNM (1974)

10. JCGM 200:2008, International vocabulary of metrology Basic and general concepts and associated terms (VIM), BIPM, IEC, IFCC, ILAC, ISO, IUPAC, IUPAP and OIML

11. JCGM 200:2008, International vocabulary of metrology Basic and general concepts and associated terms (VIM), BIPM, IEC, IFCC, ILAC, ISO, IUPAC, IUPAP and OIML, 2.43 Note

12. Key Comparison EURAMET.PR-K3.A, Luminous intensity, pilot PTB, Draft Report to release in 2010 